\title{
PREVALÊNCIA DE PARASITOSES INTESTINAIS EM USUÁRIOS DE UM HOSPITAL UNIVERSITÁRIO, SANTA CRUZ-RN, BRASIL
}

\section{PREVALENCE OF INTESTINAL PARASITOSIS IN USERS OF A UNIVERSITY HOSPITAL, SANTA CRUZ-RN, BRAZIL}

Ericlebson Cleyton da Silva Lima ${ }^{\mathrm{a}^{*}}$

Orcid: https://orcid.org/0000-0003-2577-9105

\author{
Heloisa Mara Batista Fernandes Oliveira ${ }^{\mathrm{b} *}$ \\ Orcid: https://orcid.org/0000-0001-8237-9920
}

\author{
Carlos Marcio Ponte Leon ${ }^{c^{*}}$ \\ Orcid: https://orcid.org/0000-0002-5353-8408
}

\author{
Vanessa Santos de Arruda Barbosa ${ }^{\star}$ \\ Orcid: https://orcid.org/0000-0002-0321-7163
}

erclebson_cleyton@hotmail.com ${ }^{\mathrm{a}}$, heloisambf@gmail.com ${ }^{\mathrm{b}}$, cmpleon@gmail.com $^{\mathrm{c}}$, vanessabarbosa@ufcg.edu.br ${ }^{\mathrm{d}}$ Universidade Federal de Campina Grande ${ }^{*}$, Universidade Federal do Rio Grande do Norte ${ }^{* *}$

Data de Submissão: 10/08/2019 Data de Aceite:18/12/2019

\section{RESUMO}

Introdução: Enteroparasitas são helmintos e protozoários que atingem o trato intestinal causando infecções denominadas enteroparasitoses. Tais parasitos podem provocar diversos transtornos ao hospedeiro. O presente trabalho objetivou determinar a prevalência das enteroparasitoses em usuários do Laboratório de Análises Clínicas do Hospital Universitário Ana Bezerra em Santa Cruz-RN. Metodologia: O estudo foi do tipo retrospectivo documental, no qual usou-se o banco de dados do CompLab do período de julho/2016 a julho/2017. Foram coletados os resultados dos laudos dos exames parasitológicos de fezes e variáveis epidemiológicas como: sexo, idade e área de residência. Foi utilizado o teste qui-quadrado $(\chi 2)$ e foi aceito $p<0,05$, estatisticamente significativo. Os dados foram analisados no software SPSS. Resultados: De 3.480 laudos, 32,4\% eram positivos para enteroparasitos e/ou enterocomensais. Dentre os positivos (1.129), 75,5\% estavam monoparasitados e 24,5\% bi/poliparasitados. As espécies mais prevalentes entre os monoparasitados foram Endolimax nana (76,2\%) e Giardia lamblia (12,2\%). A associação parasitária mais prevalente foi Entamoeba coli + Endolimax nana (52,8\%). Dentre os positivos prevaleceu o sexo feminino (63,5\%), faixa etária de 20-59 anos (44,5\%) e os residentes de zona urbana (77,8\%). No entanto não foi encontrada associação estatisticamente significativa entre essas variáveis. Infecções por Giardia prevaleceram em crianças (54,8\%). Nos adultos (20-59 anos) prevaleceram as infecções por Entamoeba histolytica/E.dispar (44,4\%) e os casos de bi/poliparasitismo (48,7\%). Conclusão: Os resultados indicam necessidade de implantação de medidas profiláticas eficazes e ações educacionais, principalmente nos grupos mais expostos a essas infecções.

Palavras-chave: Epidemiologia; doenças parasitárias; diagnóstico laboratorial.

\section{ABSTRACT}

Introduction: Enteroparasites are helminths and protozoa that reach the intestinal tract causing infections called enteroparasitosis. Such parasites can cause disorders to the host. The present work aimed to determine the prevalence of intestinal parasites in users of Clinical Analysis Laboratory of Ana Bezerra Universitary Hospital in Santa Cruz-RN. Metodology: The was a retrospective documentary study, using the CompLab databases from July/2016 to July/2017. The results of stoll parasitological examination and epidemiologicals variables such as sex, age and residence area, were collected. Chi-square test $(\chi 2)$ was used and $\mathrm{p}<0,05$ statistically significant, was accepted. Data were analyzed using SPSS software. The chi-square $(\chi 2)$ test as used, and was accepted $\mathrm{p}<0,05$, statistically significant. Results: Of 3.480 reports, $32,4 \%$ were positive for instestinal's parasites and/or intestinal's commensals. Among the positive one (1.129), 75,5\% was monoparasitic and $24.5 \%$ bi/poliparasitic. The most prevalent species among monoparasites were Endolimax nana (76.2\%) and Giardia lamblia (12.2\%). The most prevalent parasitic association was Entamoeba coli + Endolimax. nana (52.8\%). Among the positives results, prevailed were female (63.5\%), age range 20-59 years (44.5\%) and urban residents (77.8\%). However, no statistically significant association was found between these variables. Giardia lamblia infections prevailed in children (54.8\%). In adults (20-59 years) infections by Entamoeba hystolitica/E. dispar (44.4\%) and cases of bi/poliparasitism (48.7\%) prevailed. Conclusion: The results indicate the need to implement effective prophylactic measures and educational actions, especially in the most exposed groups to these infections.

Keywords: Epidemiology; parasitic diseases; laboratory diagnosis. 


\section{Introdução}

As parasitoses intestinais constituem um dos maiores agravos na saúde pública em todo o mundo, estando presente principalmente em países subdesenvolvidos e em desenvolvimento. 1,2. As enteroparasitoses são classificadas como doenças negligenciadas por apresentarem baixos investimentos no seu controle, na pesquisa e produção de medicamentos, fazendo com que haja uma elevada prevalência na população, principalmente naquela que vive em condições de pobreza, o que colabora para a manutenção do quadro de desigualdade. A suscetibilidade às enteroparasitoses está, muitas vezes, relacionada ao nível socioeconômico, hábitos pessoais, condições higiênicas e sanitárias, dentre outros fatores em que a população está inserida ${ }^{3,4}$.

Os enteroparasitas são helmintos e protozoários que habitam o trato intestinal. A preocupação com tais parasitas ocorre devido a uma série de patologias que estes podem causar como: ascaridíase, giardíase, teníase, amebíase, dentre outras, e ainda devido aos transtornos que podem provocar no portador, como: máabsorção, anemia, menor capacidade de trabalho; e nas crianças, baixo rendimento escolar e déficit no crescimento ${ }^{5,6}$.

$\mathrm{Na}$ maioria dos casos de patologias causadas por parasitas intestinais, o tratamento se dá de maneira facilitada, onde o problema é resolvido com administração de medicamentos via oral, os quais estão disponíveis em todas as regiões do país pelo Sistema Único de Saúde (SUS). Contudo, é indispensável a prática de ações preventivas e educativas em todos os contextos sociais no tocante às parasitoses intestinais, no que diz respeito ao manuseio, preparo, forma de armazenamento de alimentos e água a serem utilizados, além de obtenção de informações acerca desses danos à saúde da população, diminuindo assim o risco de reinfecção ${ }^{1,7}$.

Os conhecimentos adquiridos acerca da biologia, epidemiologia e prevenção de doenças causadas por agentes parasitários, do mesmo modo que a síntese de fármacos antiparasitários, fez com que alguns segmentos da população mundial fossem beneficiados com os avanços concedidos com esses conhecimentos nas últimas décadas.
Contudo, ainda restam comunidades concentradas principalmente em países subdesenvolvidos e em desenvolvimento, que constituem as periferias de um mundo globalizado, mas também montantes em países desenvolvidos, que continuam a padecer com as consequências das infecções parasitárias, particularmente aquelas que atingem o trato digestório ${ }^{8}$.

Investigações epidemiológicas que incluam a identificação das variáveis de prevalência nas diversas regiões geográficas do Brasil, visando estratégias de ação integrada no controle das mesmas, são necessárias ${ }^{4}$. Nesse contexto, o presente trabalho objetivou analisar a prevalência das enteroparasitoses na região de Santa Cruz-RN e áreas circunvizinhas, identificando as principais espécies de parasitos circulantes na região e algumas variáveis sociodemográficas associadas à infecção parasitária.

\section{Metodologia}

Os dados para realização do estudo foram coletados do banco de dados do Laboratório de Análises Clínicas do Hospital Universitário Ana Bezerra (HUAB), vinculado à UFRN e administrado pela Empresa Brasileira de Serviços Hospitalares (EBSERH). O mesmo situa-se no município de Santa Cruz, Rio Grande do Norte e é atualmente a referência de atendimento obstétrico e pediátrico no município de Santa Cruz-RN e cidades circunvizinhas?.

Foi realizado um estudo epidemiológico delineado como retrospectivo documental, em que foram analisados os resultados dos Exames Parasitológicos de Fezes (EPF) e as variáveis sociodemográficas dos usuários, registradas no banco de dados do Laboratório de Análises Clínicas do HUAB do período de julho de 2016 a julho de 2017.

Foram coletadas as seguintes variáveis constantes do laudo do EPF: idade, sexo, área de residência, resultado e metodologia do diagnóstico parasitológico. As variáveis foram apresentadas na forma de número absoluto e percentuais e foi utilizado o teste qui-quadrado $(\chi 2)$ para se verificar associação entre as variáveis. Foi aceito 
$\mathrm{p}<0,05$, estatisticamente significativo, como critério para rejeição das hipóteses de nulidade. Os dados foram analisados no programa estatístico Statistical Package for the Social Sciences (SPSS), versão 24.0.

O projeto de pesquisa foi submetido ao Comitê de Ética em Pesquisa (CEP) da Universidade Federal do Rio Grande do Norte, sendo aprovado com parecer $n^{\circ} 2.413 .829$.

\section{Resultados}

No Laboratório de Análises Clínicas do HUAB, realizou-se, no período estudado, 3.480 EPFs, em que a prevalência de infectados por enteroparasitas/enterocomensais foi de $32,4 \%$.

Dentre os 1.129 analisados com resultados positivos, $75,5 \%$ estavam monoparasitados, $24,5 \%$ estavam bi/poliparasitados, ou seja, infectados por duas ou mais espécies parasitárias. Os enteroparasitas/enterocomensais mais prevalentes nos monoparasitados foram: Endolimax nana (76,2\%), Giardia lamblia (12,2\%), Entamoeba coli $(7,4 \%)$ (Tabela 1$)$.

Tabela 1 - Prevalência das espécies de enteroparasitos/enterocomensais em indivíduos monoparasitados em usuários do Laboratório de Análises Clínicas do HUAB, Santa Cruz, 2016 a 2017.

\begin{tabular}{lll}
\hline Espécies & N & \% \\
Endolimax nana & 649 & 76,2 \\
Giardia lamblia & 104 & 12,2 \\
Entamoeba coli & 63 & 7,4 \\
Entamoeba hystolitica/E. dispar & 18 & 2,1 \\
Iodamoeba butschilii & 12 & 1,4 \\
Ascaris lumbricoides & 4 & 0,5 \\
Strongyloides stercoralis & 2 & 0,2 \\
\hline Total & 852 & 100 \\
\hline
\end{tabular}

Fonte: Dados da pesquisa

Nos casos de biparasitismo (20,6\%) observou- + Endolimax nana (52,8\%) (Tabela 2). se maior prevalência da associação Entamoeba coli

Tabela 2 - Prevalência das espécies de enteroparasitos/enterocomensais em indivíduos biparasitados em usuários do Laboratório de Análises Clínicas do HUAB, Santa Cruz, 2016 a 2017.

\begin{tabular}{lll}
\hline Espécies & $\mathbf{N}$ & $\mathbf{\%}$ \\
Entamoeba coli + Endolimax nana & 124 & 52,8 \\
Endolimax nana + Giardia lamblia & 43 & 18,3 \\
Endolimax nana + Iodamoeba butschilii & 22 & 9,4 \\
Endolimax nana + Entamoeba hystolitica & 18 & 7,6 \\
Entamoeba coli + Entamoeba hystolitica & 18 & 7,6 \\
Entamoeba coli + Iodamoeba butschilii & 5 & 2,1 \\
\hline
\end{tabular}




\begin{tabular}{lll}
\hline Espécies & $\mathbf{N}$ & $\mathbf{\%}$ \\
Ascaris lumbricoides + Giardia lamblia & 2 & 0,9 \\
Entamoeba coli + Giardia lamblia & 2 & 0,9 \\
Iodamoeba butschilii + Giardia lamblia & 1 & 0,4 \\
\hline Total & 235 & 100 \\
\hline
\end{tabular}

Fonte: Dados da pesquisa

No poliparasitismo $(3,9 \%)$, observou-se maior percentual $(26,2 \%)$ na associação das espécies
Endolimax nana, Entamoeba coli e Entamoeba histolytica/E. dispar (Tabela 3).

Tabela 3 - Prevalência das espécies de enteroparasitos/enterocomensais em indivíduos poliparasitados em usuários do Laboratório de Análises Clínicas do HUAB, Santa Cruz, 2016 a 2017.

\begin{tabular}{lll}
\hline Espécies & $\mathbf{N}$ & $\mathbf{\%}$ \\
E. coli + E. nana + E. histolytica & 11 & 26,3 \\
E. nana + I. butschilii + E. histolytica & 10 & 23,8 \\
E. nana + E. coli + I. butschilii & 9 & 21,4 \\
E. nana + E. coli + G. lamblia & 4 & 9,5 \\
E. nana + E. coli + I. butschilii + E. histolytica & 4 & 9,5 \\
E. nana + E. coli + E. histolytica & 3 & 7,1 \\
E. nana + I. butschilii + G. lamblia & 1 & 2,4 \\
\hline Total & 42 & 100 \\
\hline
\end{tabular}

Fonte: Dados da pesquisa

As espécies patogênicas com maior prevalência foram Giardia lamblia (12,2\%) e Entamoeba hystolitica/E. dispar (2,1\%). De acordo com os grupos etários o mais infectado por Giardia lamblia foi o infantil (0 a 09 anos), representando 54,8\% dos infectados e por Entamoeba hystolitica/E. dispar, os adultos (20 a 59 anos) com $44,4 \%$.

A metodologia de análise utilizada em $100 \%$ dos laudos foi a técnica de sedimentação espontânea.
O perfil da população analisada foi categorizado segundo o sexo, idade e local de domicílio. A tabela 3 mostra o percentual de indivíduos parasitados de acordo com o sexo. Observou-se que dentre os positivos, $63,5 \%$ eram do sexo feminino. Não foi observada associação estatística entre as variáveis $(\mathrm{p}=0,825)$ (tabela 4$)$. 
Tabela 4 - Distribuição da prevalência de enteroparasitoses/enterocomensais segundo o sexo em usuários do Laboratório de Análises Clínicas do HUAB, Santa Cruz, 2016 a 2017.

\begin{tabular}{|c|c|c|c|c|c|c|c|}
\hline \multirow{3}{*}{ Categoria } & \multicolumn{4}{|c|}{ Resultado } & \multicolumn{2}{|c|}{ Total } & \multirow[t]{3}{*}{ Valor $\mathrm{p}$} \\
\hline & \multicolumn{2}{|c|}{ Positivo } & \multicolumn{2}{|c|}{ Negativo } & \multirow[b]{2}{*}{$\mathrm{N}$} & \multirow[b]{2}{*}{$\%$} & \\
\hline & $\mathrm{N}$ & $\%$ & $\mathrm{~N}$ & $\%$ & & & \\
\hline \multicolumn{8}{|l|}{ Sexo } \\
\hline Masculino & 412 & 36,5 & 867 & 36,9 & 1279 & 36,8 & \multirow{2}{*}{0,825} \\
\hline Feminino & 717 & 63,5 & 1484 & 63,1 & 2201 & 63,2 & \\
\hline Total & 1129 & 100 & 2351 & 100 & 3480 & 100 & \\
\hline
\end{tabular}

Fonte: Dados da pesquisa

Quanto à prevalência por grupo etário, verificou-se que adultos (20 a 59 anos) foram os mais parasitados $(44,5 \%)$, seguidos de crianças (0 a 9 anos) $25,5 \%$, idosos (60 anos ou mais) $16,7 \%$ e adolescentes da faixa etária de 10 a 19 anos (13,3\%). Não foi observada associação estatisticamente significativa entre os grupos etários $(\mathrm{p}=0,066)$ (Tabela 5).

Tabela 5 - Associação entre bi/poliparasitados de acordo com a faixa etária em usuários do Laboratório de Análises Clínicas do Hospital Universitário Ana Bezerra (HUAB), Santa Cruz, 2016 a 2017.

\begin{tabular}{cccccccc}
\hline & \multicolumn{9}{c}{ Resultado } & \multicolumn{2}{c}{ Total } & Valor p \\
\hline \multirow{3}{*}{ Categoria } & \multicolumn{2}{c}{ Bi/Poliparasitado } & Monoparasitado & & \\
\cline { 2 - 6 } & $\mathrm{N}$ & $\%$ & $\mathrm{~N}$ & $\%$ & $\mathrm{~N}$ & $\%$ \\
\hline
\end{tabular}

\section{Faixa etária}

\begin{tabular}{cccccccc}
0 a 9 anos & 54 & 19,5 & 234 & 27,5 & 288 & 25,5 & \\
10 a 19 anos & 37 & 13,4 & 113 & 13,3 & 150 & 13,3 & 0,062 \\
20 a 59 anos & 135 & 48,7 & 367 & 43,1 & 500 & 44,5 & \\
60 anos ou mais & 51 & 18,4 & 138 & 16,2 & 189 & 16,7 & \\
\hline Total & 277 & 100 & 852 & 100 & 1129 & 100 \\
\hline
\end{tabular}

Fonte: Dados da pesquisa.

Analisando-se a associação entre bi/ poliparasitados e faixas etárias, verifica-se maior prevalência de bi/poliparasitados na faixa etária de 20 a 59 anos (48,7\%). Porém, não houve associação estatisticamente significativa $(\mathrm{p}=0,062)$.
Quanto ao local de moradia da população estudada, entre os 1.129 resultados positivos, $77,8 \%$ dos positivos eram domiciliados na zona urbana. Estatisticamente não houve diferença significativa entre eles $(\mathrm{p}=0,793)$ (Tabela 6). 
Tabela 6 - Distribuição da prevalência de enteroparasitoses/enterocomensais segundo a zona de domicílio em usuários do Laboratório de Análises Clínicas do HUAB, Santa Cruz, 2016 a 2017.

\begin{tabular}{lccccccc}
\hline & \multicolumn{9}{c}{ Resultado } & \multicolumn{2}{c}{ Total } & Valor p \\
\hline \multirow{2}{*}{ Categoria } & Positivo & \multicolumn{2}{c}{ Negativo } & & & \\
\cline { 2 - 9 } Domicílio & $\mathrm{N}$ & $\%$ & $\mathrm{~N}$ & $\%$ & $\mathrm{~N}$ & $\%$ & \\
Zona urbana & 878 & 77,8 & 1819 & 77,4 & 2697 & 77,5 & 0,793 \\
Zona rural & 251 & 22,2 & 532 & 22,6 & 783 & 22,5 & \\
\hline Total & 1129 & 100 & 2351 & 100 & 3480 & 100 & \\
\hline
\end{tabular}

Fonte: Dados da pesquisa

\section{Discussão}

Dos 3.480 resultados analisados no estudo, $32,4 \%$ eram positivos para, pelo menos, uma espécie de enteroparasito e/ou enterocomensal. Estudos apontam resultados variados em relação a essa prevalência, de acordo com a região e a população pesquisada.

Prevalências parecidas foram encontradas em Chapadinha - MA, em que, das 3.993 amostras fecais analisadas, $33,2 \%$ estavam infectadas com enteroparasitas/enterocomensais ${ }^{10}$; na Mesorregião do Oeste Potiguar, que compreende os municípios norteriograndenses de Areia Branca, Baraúna, Governados DixSept Rosado, Grossos, Mossoró, Pau dos Ferros e Upanema, dos 8.456 registros de resultados de EPF, 33,1\% estavam infectadas ${ }^{11}$; e em Santa Luzia - PB, onde $30 \%$ dos 1.000 resultados analisados eram positivos ${ }^{12}$.

Prevalências inferiores foram encontradas em Bom Jesus dos Perdões - SP, em que 15,7\% das 2.524 amostras analisadas eram positivas para espécies parasitárias/comensais ${ }^{13}$; e, no município de Macapá - AP, em que dos 9.842 resultados de EPF realizados, a prevalência foi de $13,6 \%$ de resultados positivos ${ }^{14}$.

Taxas de prevalências superiores foram encontradas no município de Parelhas - RN, com 45,2\% dos 1.279 resultados de $\mathrm{EPF}^{15}$; e, no município de Conde - PB que apresentou taxa de positividade de $42,1 \%$ dos 1.759 resultados analisados ${ }^{16}$.
Dos 1.129 resultados positivos, 20,6\% apresentaram infecção por duas espécies e 3,9\% por mais de duas (poliparasitismo). Essa situação pode ser favorecida por uma maior exposição aos fatores de risco, como condições sanitárias precárias e, principalmente, pelo fato de o mecanismo de transmissão de vários parasitas intestinais ser igual ${ }^{17,18}$. Prevalência maior foi vista em Chapadinha - MA, onde das 1.301 amostras positivas, $24,1 \%$ se apresentavam poliparasitadas ${ }^{10}$. Índices mais baixos de poliparasitismo foram observados em Cumari - GO, em que dos 373 casos positivados, $1,1 \%$ era poliparasitado ${ }^{19}$ e no município de Maria Helena - PR que apresentou $2,3 \%$ de poliparasitados, dentre os 69 positivos $^{20}$. No município de Parelhas - RN foi observado um alto índice de poliparasitismo em comparação aos resultados obtidos no presente estudo, em que $41 \%$ dos 524 positivos estavam poliparasitados ${ }^{15}$.

Observa-se, portanto, que as taxas de prevalência dos municípios nordestinos, citados acima, foram iguais ou superiores a $30 \%$ de positividade para enteroparasitos e superiores a $20 \%$ para as taxas de bi/poliparasitismo, em comparação com os municípios das outras regiões brasileiras. Essas diferenças regionais podem ter relação com fatores climáticos relacionados às condições de temperatura e umidade, que permitem a manutenção do peridomícilio como foco de parasitoses; cobertura do sistema público de abastecimento de água e de coleta de esgoto; expansão das áreas de favelização, com moradias precárias e aglomerações humanas; precariedade de serviços de coleta de lixo; hábitos alimentares 
e higiênicos da população, além de renda e nível educacional ${ }^{4,7}$.

$\mathrm{Na}$ distribuição da frequência de enteroparasitas/enterocomensais que foram encontradas em indivíduos monoparasitados, as maiores prevalências foram: Endolimax nana (76,2\%), Giardia lamblia (12,2\%), Entamoeba coli $(7,4 \%)$ e Entamoeba hystolitica/E. dispar (2,1\%). Destaca-se que a infecção pelos enterocomensais Endolimax nana e Entamoeba coli apesar de não apresentarem importância clínica, tem valor epidemiológico, pois suas transmissões estão ligadas à contaminação por material fecal em alimentos e água de consumo ${ }^{21,22}$. Embora estes parasitas não sejam considerados patogênicos, é importante ressaltar esses resultados, uma vez que esse índice pode ser usado como parâmetro para medir o grau de contaminação fecal-oral em que os indivíduos estudados estão expostos ${ }^{20}$.

Estudos de prevalência apresentam semelhança em relação ao maior número de indivíduos infectados por Endolimax nana como em Bom Jesus dos Perdões - SP, em que 44,7\% dos 396 estavam com essa espécie ${ }^{13}$ e em Maria Helena $\mathrm{PR}$, em que a mesma foi encontrada em $40,5 \%$ dos 69 positivos $^{20}$.

Nessa pesquisa, o parasita patogênico mais observado na população em geral foi Giardia lamblia (12,2\%), tendo maior prevalência na faixa etária de 0 a 9 anos (54,8\%). Essa espécie patogênica foi a mais prevalente em estudos realizados em Iretama - PR, em que se observou frequência de $31,2 \%$ em 32 escolares estudados ${ }^{23}$; em Chapadinha - MA, das 322 crianças, de 0-10 anos, infectadas $31,3 \%$ eram por Giardia lamblia ${ }^{10}$; e em Cumarí - GO a prevalência foi de $42,4 \%$ nessa mesma faixa etária, dos 346 infectados por Giardia lamblia ${ }^{19}$.

A infecção por Giardia na infância é preocupante do ponto de vista clínico por estar relacionada a casos de diarreia grave e deficiências na absorção de nutrientes ${ }^{4}$.

No presente estudo, $44,4 \%$ das infecções por Entamoeba hystolitica/E. dispar, foram em adultos (20-59 anos). Em Chapadinha - MA, também observaram alto percentual, em que $56,6 \%$ dos 496 infectados por essas espécies estavam na faixa etária de 21 a $60 \operatorname{anos}^{10}$.
A prevalência de infecção para Ascaris lumbricoides foi de apenas $0,5 \%$, porém a infecção por essa espécie é preocupante, pois os parasitos na forma larvária podem causar danos hepáticos e pulmonares, devido suas migrações; e os adultos, danos intestinais por ação mecânica, desnutrição, por ação espoliadora, ação tóxica de antígenos parasitários e até fazer localizações ectópicas nas infecções maciças ${ }^{24}$.

A espécie patogênica com menor prevalência foi a Strongyloides stercoralis (0,2\%). De certa forma, a não realização de métodos de diagnóstico baseados no hidrotermotropismo das larvas de $S$. stercoralis, teste específico para este helminto, não permite avaliar a real prevalência dessa parasitose na população ${ }^{25,26}$.

Dentre os casos de biparasitismo, a associação entre os enterocomensais Endolimax nana e Entamoeba coli foi a mais prevalente, sendo observada em 52,8\% dos casos. Essa mesma associação foi visualizada em Maria Helena $\mathrm{PR}$, em $50 \%$ dos biparasitados $(\mathrm{N}=14)^{20}$, em Bom Jesus dos Perdões - SP, em 17,9\% do total de 28 biparasitados $^{13}$; e na Mesorregião do Oeste Potiguar, em que $34 \%$ dos 494 biparasitados apresentaram coinfecção por essas mesmas espécies ${ }^{11}$.

No poliparasitismo, com referência a associações com três parasitas, obteve-se uma maior associação entre Endolimax nana, Entamoeba coli e Entamoeba hystolitica/E.dispar (25\%), os primeiros são protozoários comensais e o último, parasita patogênico. Em levantamento realizado em sete municípios da Mesorregião Potiguar, a coinfecção por essas espécies foi encontrada em $62,5 \%$ dos 80 infectados com três espécies, sendo a mais prevalente ${ }^{11}$.

O Laboratório de Análises Clínicas do HUAB, por questões financeiras e de demandas, utiliza na rotina laboratorial apenas o método de sedimentação espontânea ou Hoffman, Pons e Janer (HPJ), no processamento das amostras fecais para diagnóstico de enteroparasitas/enterocomensais. Esse método identifica cistos de protozoários e ovos pesados de helmintos, dificultando assim o diagnóstico de infecção por algumas espécies que só são evidenciadas por técnicas especiais ${ }^{13,20}$. Em estudos comparativos entre os métodos de diagnóstico por técnicas de centrífugo-flutuação 
em sulfato de zinco (Faust et cols.), HPJ e Faust et cols. + HPJ, foi evidenciada a maior sensibilidade do método de Faust et cols. para cistos de protozoários. Observaram ainda que a utilização do método de Faust et cols. como método complementar ao HPJ, melhora significativamente os resultados do diagnóstico ${ }^{27,28}$. Com o interesse em obter qualidade nos resultados de exames parasitológicos, aconselha-se a utilização de mais de um método, sendo esse processo dificultado pela não adesão dos órgãos governamentais, pelo elevado custo ${ }^{29}$.

Observou-se que $63,2 \%$ do total das amostras analisadas eram de indivíduos do sexo feminino, o que pode ser explicado devido aos dados coletados serem obtidos de uma maternidade. Também foi o sexo feminino o que apresentou maior prevalência de infectados $(63,5 \%)$, porém estatisticamente não ficou demonstrada diferença significativa $(\mathrm{p}=0,944)$, podendo concluir que a positividade desses casos não teve dependência em relação aos sexos avaliados. Em comparação a outros estudos, esse resultado é compatível com os obtidos em Ferreira Gomes - AP, em que das 130 amostras positivas, $64,6 \%$ eram do sexo feminino ${ }^{22}$; em Assis - SP, em que 59\% das 254 amostras infectadas, eram provenientes do sexo feminino ${ }^{30}$; em Parelha - $\mathrm{RN}$, em que se observou 529 resultados positivos, sendo $54 \%$ provenientes do sexo feminino ${ }^{15}$; e em Pombal - PB, em que 70,5\% das 910 amostras positivas eram do sexo feminino ${ }^{31}$, nenhum desses observando significância estatística.

De acordo com as faixas etárias, a maior prevalência de infectados se deu no grupo que compreende as idades de 20 a 59 anos $(43,9 \%)$ e apesar dessa alta prevalência, não houve associação estatisticamente significativa $(p=0,066)$. Análise realizada em Ibiassucê - BA, demonstra similaridade com o presente estudo, onde o grupo com maior prevalência foi 16-59 anos, observando-se 54,8\% dos 683 infetados $^{32}$.

Do total de analisados, $77,5 \%$ dos indivíduos residiam na zona urbana, o que pode ser explicado devido à facilidade de acesso ao serviço de saúde. Os domiciliados na zona urbana também foram os mais infectados $(77,8 \%)$ quando comparados aos da zona rural $(22,2 \%)$, porém, não se observou significância estatística entre eles $(p=0,793)$. Em comparação com esse resultado, no município de Parelhas - RN, foi observado que entre os indivíduos infectados (524), 84,5\% eram oriundos da zona urbana, sendo demonstrado uma significância estatística $(\mathrm{p}=0.0001)$, e fator de risco, 2,19 vezes maior para os indivíduos da zona urbana $^{15}$.

\section{Conclusões}

A frequência de indivíduos infectados foi de $32,4 \%$, tendo como maior prevalência as espécies de enterocomensais: E. nana e E. coli, que são marcadoras da contaminação fecal-oral. Embora a maior prevalência de parasitados tenha sido do sexo feminino, da faixa etária de 20-59 anos e residentes da área urbana, não ficou demonstrada associação estatisticamente significativa entre essas variáveis. $\mathrm{O}$ parasita intestinal patogênico mais frequente foi a Giardia lamblia, sendo mais prevalente nas crianças.

A alta prevalência de pessoas infectadas em Santa Cruz-RN reflete a realidade acerca das condições de vida apresentadas no município, com precariedade sanitária, e baixas condições socioeconômicas e educacionais em que vive grande parte da população. Diante desses resultados deve-se considerar a necessidade de se instituir medidas diagnósticas, terapêuticas e profiláticas como expansões do sistema de saneamento básico para todas as regiões do município e implementação de educação sanitária na população.

Condições apropriadas de saneamento básico, ações de educação higiênico-sanitária, principalmente para as mulheres adultas e crianças, e o emprego correto de medicamentos de fácil acesso para o tratamento das enteroparasitoses, são fatores essenciais para aumentar as condições de saúde da população.

O estudo apresentado necessita de continuidade para aprimorar parâmetros que possam indicar mais fatores que colaborem com a alta prevalência das enteroparasitoses na população. Para isso, necessita-se fazer a coleta de outras variáveis epidemiológicas dos usuários do laboratório de análises clínicas, para que se possa mapear fatores de risco envolvidos na infecção parasitária. 


\section{Referências}

1. Basso RMC, Silva-Ribeiro RT, Soligo DS, Ribacki SI, Callegari-Jacques SM, Zoppas BCA. Evolução da prevalência de parasitoses intestinais em escolares em Caxias do Sul, RS. Rev Soc Bras Med Trop. 2008; 41(3): 263-268.

2. Menezes VFP, Medeiros NS, Carolini D. Prevalência de enteroparasitoses em escolares: Uma revisão do perfil encontrado em diferentes regiões do Brasil. Rev Bras Multidisc. 2012; 15(2):7-18.

3. Uchoa CMA, Albuquerque MC, Carvalho FM, Falcão AO, Silva P, Bastos OMP. Parasitismo Intestinal em crianças e funcionários de creches comunitárias na cidade de Niterói- RJ, Brasil. Rev Patol Trop. 2009; 38(4):267-278.

4. Andrade EC, Leite ICG, Oliveira Rodrigues V, Cesca MG. Parasitoses intestinais: Uma revisão sobre seus aspectos sociais, epidemiológicos, clínicos e terapêuticos. Rev APS. 2010; 13(02):231-240.

5. Pittner E, Moraes IF, Sanches HF, Trincaus MR, Raimondo ML, Monteiro MCM. Enteroparasitoses em crianças de uma comunidade escolar na cidade de Guarapuava/PR. Rev Salus. 2007; 1(1):97-100.

6. Santiago AC, Gagliane, LH. Estudo da prevalência de enteroparasitas em areia de praia no município de São Vicente - SP - Brasil. Rev UNILUS Ens Pesq. 2011; 8(15):5-19.

7. Barbosa LA, Sampaio ALA, Melo ALA, Macedo APN, Machado MFAS. A educação em saúde como instrumento na prevenção de parasitoses. Rev Bras Promo Saúde. 2009, 22(4):272-278.

8. Coradi ST. Epidemiologia das parasitoses intestinais e caracterização genotípica de isolados de Giardia duodenalis de escolares do município de Pratânia, estado de São Paulo. 2010. 138 f. Tese (doutorado) - Universidade Estadual Paulista, Faculdade de Medicina de Botucatu, 2010.

9. EBSERH. Empresa Brasileira de Serviços Hospitalares. Hospital Universitário Ana Bezerra - Estrutura Assistencial. Disponível em: http://www2.ebserh.gov. br/web/huab-ufrn/nossa-historia. Acesso em: 09 de abr. 2018 .

10. Silva FS, Paulo ADC, Braga CMM, Almeida RJ, Galvão VP. Frequência de parasitos intestinais no município de Chapadinha, Maranhão, Brasil. Rev Patol Trop. 2010, 39(1):63-68.

11. Oliveira CHB. Frequência de enteroparasitoses em usuários do serviço público de saúde na Mesorregião do Oeste Potiguar. 2013. 47 f. Dissertação (Mestrado em Saúde Pública) - Universidade Federal do Rio Grande do Norte, Natal, 2013.
12. Lima EQ, Santos MT, Siqueira RR, Medeiros Filho F, Pontes Filho RN. Prevalence of intestinal parasites in the human population of the city Santa Luzia - State of Paraíba, Brazil. J Parasitol Vector Biol. 2016, 8(9):86-91.

13. Lodo M, Oliveira CGB, Fonseca ALA, Caputto LZ, Packer MLT, Valentin VE, Fonseca FLA. Prevalência de Enteroparasitoses em Município do Interior Paulista. Rev Bras Crescimento Desenvolv Hum. 2010, 20(3):769- 777.

14. Ferraz RRN, Barnabé AS, Porcy C, D’eça Júnior A, Feitosa T, Figueiredo PM. Intestinal parasitosys and reduced Gini index in Macapá (AP) and Timon (MA), Brazil. Cad Saúde Colet. 2014, 22(2):173-176.

15. Macedo MFS, Sisenando HAAACN, Bezerra Neto MM, Saturtino ACRD. Prevalência de enteroparasitoses, no município de Parelhas, Rio Grande do Norte, Brasil. Infarma. 2008, 20(3):35-40.

16. Oliveira Filho AA, Habrantes HFL, Fernandes HMB, Viana WP, Pinto MAS, Cavalcanti AL, Freitas FIS. Perfil enteroparasitológico dos habitantes de uma cidade do Nordeste do Brasil. Rev Bras Clin Med. 2012, 10(3):179. 182.

17. Zaiden MF, Santos BO, Cano MAT, Nascif Júnior IA. Epidemiologia das parasitoses intestinais em crianças de creches de Rio Verde - GO. RMRP. 2008, 41(2):182-187.

18. Andrade EC, Leite ICG, Vieira MT, Abramo C, Tibiriçá SHC, Silva PL. Prevalência de parasitoses intestinais em comunidade quilombola no Município de Bias Fortes, Estado de Minas Gerais, Brasil, 2008. Epidemiol Serv Saúde. 2011, 20(3):337-344.

19. Borges WF, Marciano FM, Oliveira HB. Parasitos intestinais: elevada prevalência de giardia lamblia em pacientes atendidos pelo serviço público de saúde da região sudeste de Goiás, Brasil. Rev Patol Trop. 2011 40(2):149-157.

20. Santos AS, Merlini LS. Prevalência de enteroparasitoses na população do município de Maria Helena, Paraná. Ciênc Saúde Col. 2010, 15(3):899-905.

21. Pereira MF, Coelho FAZ, Marson FG, Capuano DM, Kanamura HY. Ocorrência de enteroparasitos e comensais em crianças do ensino fundamental no município de Pindamonhangaba, SP. Brasil. Rev Biociênc. 2011, 17(1):40-49.

22. Martins DM, Cardoso KCI, Couto AARD. Estudo da prevalência de enteroparasitoses no município de Ferreira Gomes/AP após a enchente em 2011. Biota Amazôn. 2014, 4(3):15-24.

23. Mamus CNC, Moitinho ACC, Grube CC, Melo EM, Weiler EB, Abreu CA, Beltrão L, Soares PB, Beltrame S, Ribeiro S, Aleixo DL. Enteroparasitoses em um centro de educação infantil do município de Iretama/PR. Sabios Rev. Saúde e Biol. 2008, 3(2):39-44. 
24. Lamberton PHL, Jourdan PM. Human Ascariasis: Diagnostics Update. Curr Trop Med Rep. 2015, 2(4):189200.

25. Machado ER, Souza TS, Costa-Cruz JM. Strongyloides stercoralis and other enteroparasites in individuals of rural area of Uberlândia, Minas Gerais state, Brazil. Rev Patol Trop. 2010, 39(2):115-122.

26. Bosqui LR, Pereira VL, Lucas BB, Dalavedo GA, Santos NCC, Freire ACARB, Luis NCO, Murad VA, Custódio LA, Almeida RS, Conchon-Costa I, Pavanelli WR, Costa IN. Occurrence of Strongyloides stercoralis and other intestinal parasites in individuals from the Northern Parana region. Biosaúde. 2014, 16(1):8-18.

27. Garcia JGD, Simões MJS, Alvarenga VL. Avaliação de diferentes métodos no diagnóstico laboratorial de Giardia lamblia. Rev Ciênc Farm Bás Aplic. 2006, 27(3):253-258.

28. Bica VC, Dillenburg AF, Tasca T. Diagnóstico laboratorial da giardiose humana: comparação entre as técnicas de sedimentação espontânea em água e de centrífugoflutuação em solução de sulfato de zinco. Rev HCPA. 2011. v. 31(1): p. 39-45.

29. De Carli GA. Parasitologia clínica: seleção de métodos e técnicas de laboratório para o diagnóstico das parasitoses humanas. $2^{\mathrm{a}}$ ed. São Paulo: Atheneu; 2011.

30. Frei F, Juncansen C, Ribeiro-Paes JT. Levantamento epidemiológico das parasitoses intestinais: viés analítico decorrente do tratamento profilático. Cad Saúde Pública. 2008, 24(12):2919-2925.

31. Lima EQ, Costa ES, Siqueira RR, Medeiros Filho F, Pontes Filho RN. Prevalence of intestinal parasites of the human population in the City of Pombal-PB, Brazil. J. Public Health Epidemiol. 2016, 8(12):343-350.

32. Matos MA, Cruz ZV. Prevalência das parasitoses intestinais no município de Ibiassuce- BA. Rev Edu Meio Amb Saúde. 2012, 5(1):64-71.

\section{Como citar este artigo:}

Lima ECS, Oliveira HMBF, Leon CMP, Barbosa VSA. Prevalência de parasitoses intestinais em usuários de um hospital universitário, Santa Cruz-RN, Brasil. Rev. Aten. Saúde. 2020; 18(63): 21-30. 